\section{Addressing future challenges for cancer services: part II}

\author{
Jane Maher ${ }^{*, 1} \&$ Gina Radford ${ }^{2}$
}

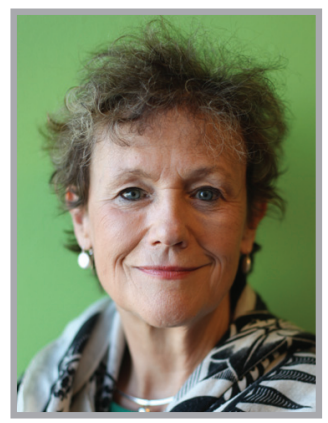

Jane Maher \& Gina Radford speak to Gemma Westcott, Commissioning Editor

Jane Maher has been Macmillan's Chief Medical Officer since 1999 and now shares the role as Joint Chief Medical Officer with general practitioner Rosie Loftus, reflecting the growing need for specialists and generalists to work more effectively together. She has been an National Health Service (NHS) improvement clinical leader for over 10 years and is a Consultant Clinical Oncologist at Mount Vernon Cancer Centre and Hillingdon Hospital where she has worked for more than 20 years, during which she helped develop nonsurgical oncology services in five district general hospitals. She is a senior Clinical Lecturer at University College London and Visiting Professor in Cancer and Supportive Care at the Centre for Complexity Management at the University of Hertfordshire. Jane chaired the Maher Committee for the Department of Health in 1995, led the UK National Audit of Late Effects Pelvic Radiotherapy for the Royal College of Radiologists (RCR) in 2000 and, most recently, chaired the National Cancer Survivorship Initiative Consequences of Treatment work stream. She co-founded one of the first Cancer Support and Information services in the UK, winning the Nye Bevan award in 1992 and there are now more than 60 units based on this model. She is a member of the Older People and Cancer Clinical Advisory Group. She has written more than 100 published articles and is a UK representative for cancer survivorship in Europe and advises on cancer survivorship programs in Denmark and Canada.

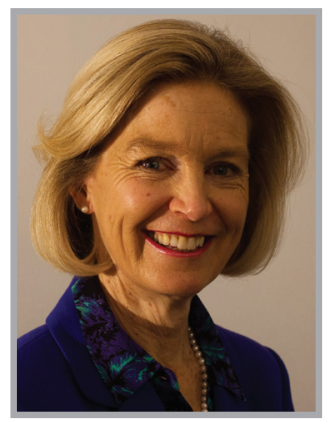

Gina Radford is Deputy Chief Medical Officer for England, a post she took up in January 2015. Prior to that, she has held a number of roles in public health, at local and regional level. Most recently she was Centre Director for Anglia and Essex for Public Health England, and as a part of that role helped lead nationally on the public health response to Ebola. She was until very recently Chair of one of the NICE public health advisory committees. She has previously worked on a number of national projects, including leading the Department of Health's response to the Shipman Enquiry, undertaking a review of specialist public health for CMO Scotland, chairing a national short life working group looking at the issue of making difficult decisions in NHS Scotland, and undertaking the evaluation of the first pilot (regional bowel cancer detection pilot) for the Be Clear on Cancer National Awareness and Early Diagnosis campaign, on behalf of the Department of Health and Cancer Research UK. Outside work, Gina is a Licensed Lay Minister in the Church of England, and is training to be ordained. She enjoys riding, walking the somewhat aging dog, reading and is the village duck warden!

'Macmillan Cancer Support, 89 Albert Embankment, London, SE1 7UQ, UK

2Department of Health, 79 Whitehall, SW1A 2NS, UK

*Author for correspondence: jmaher@macmillan.org.uk

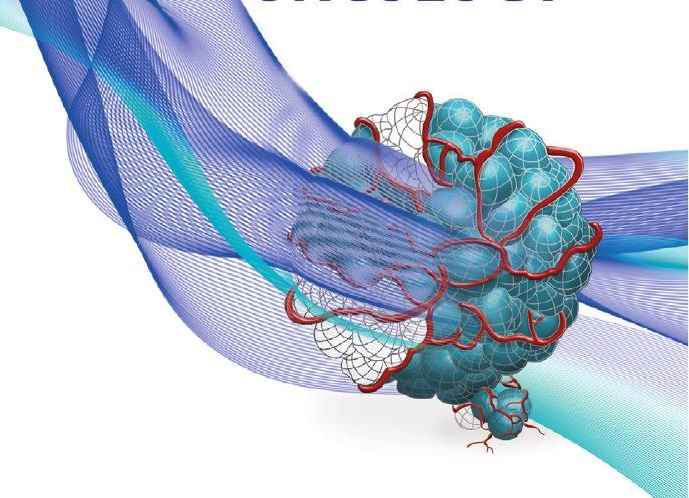

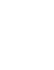




\section{KEYWORDS}

- cancer alliances • cancer cost $\bullet$ cancer registries -cancer services • cancer strategy for England

- community care

- Independent Cancer Taskforce $\bullet$ multidisciplinary team $\bullet$ public health

First draft submitted: 24 November 2015; Accepted for publication: 1 December 2015; Published online: 5 February 2016

\section{Q Organizational infrastructure is vital} for coordinating cancer care. How can we streamline clinical pathways to ensure that patients get better access \& shorter waiting times?

\section{- Gina Radford}

The model of multidisciplinary team working has revolutionized our approach to cancer treatment over the past 15 years. Multidisciplinary teams have also delivered a number of improvements in the quality of care and patient outcomes. They are seen as the 'gold standard' in terms of cancer patient management and have made a substantial contribution in reducing variation in access to treatment.

However, like all parts of the service, they are under growing pressure from increased demand and insufficient support, and are not operating as effectively as they could. They need to be supported to streamline their processes and identify opportunities to reform where possible. With this in mind, the Taskforce's report has several recommendations which seek to achieve this.

\section{- Jane Maher}

One of the recommendations in the Cancer Strategy for England is the creation of commissioning partnerships called Cancer Alliances. Macmillan is fully behind this recommendation as we believe that for people to receive high-quality treatment and services in a coordinated way, we need to see patients, experts, care providers and commissioners working together. Cancer Alliances will bring all the right people together, locally driving improvement through collaboration, and making sure that services meet the needs of local people. We want to see Cancer Alliances that work within existing health systems, making sure that in every part of the country the standard of cancer care is the best it can be.

Another recommendation in the Cancer Strategy is the implementation of stratified follow-up pathways, initially for breast cancer and then for at least two other cancer types by 2020 . Stratified pathways allow patients with the most complex care needs to be given access to all of the intensive care and support they need, while people who require fewer follow-up appointments are supported to self-manage their condition. This can reduce hospital visits that add no value, provide more personalized care, and enable resources to be refocused on rehabilitation and recovery. Pilots also indicate that these interventions are at least cost neutral.

Similar steps are also needed in the rest of the UK. Cancer services in Wales, for example, are undergoing a reorganization as current structures are not fit for purpose. We have to make sure the new structures are fully integrated and have robust governance processes.

Q How can a drive for more community-based care improve services \& prevent future problems?

- Jane Maher

Community and primary-based care is particularly important for people living with both cancer and another long-term condition, those with incurable but treatable cancer and those living with long-term consequences of either their cancer or its treatment. We also know that people with cancer have significant social care needs, particularly at the end of life but also at all stages of the pathway. In many cases, this lack of formal care at home leads to potentially avoidable A\&E visits. Improving care at home or in the community will help more people to die in the place of their choosing as well as supporting those with complex needs throughout their care.

\section{- Gina Radford}

Community-based care is a very important element in improving services, preventing future problems and promoting greater integration across the whole cancer pathway. In terms of reducing the growth in the number of cancer cases, we could do more to ensure that people are engaging with health and community services early enough to shift focus onto preventing rather than treating disease. With regards to treatment, one model being developed in several forms is that of delivery in community settings, for example, nurses from a secondary care provider delivering chemotherapy in GP premises.

The majority of patients prefer receiving chemotherapy closer to home where possible. Of course, it is imperative to clarify which drugs are safe enough to be delivered in community settings, and at what doses, for these models to be rolled out more broadly. Patient safety and management of side effects needs to be paramount. 
The new cancer strategy has a recommendation for NHS England on encouraging the delivery of chemotherapy in community settings. It also has other recommendations relevant to community services, relating to community pharmacy and community oncology nurse services among others.

\section{Q Medical technology will continue to} advance but will the timescale over which these developments occur be an issue?

\section{- Gina Radford}

We believe that patients should have access to the latest diagnostic tests and best evidence-based treatments available. Much progress has already been made in this regard - The Bowel scope screening has now been rolled out to nearly $80 \%$ of centers, exceeding the target of $60 \%$, and we are on track to open all centers in England by the end of 2016. Over 75,000 55-year-olds have been screened so far. With reference to intensitymodulated radiotherapy (IMRT), a more precise form of radiotherapy with fewer side effects, nationally, $37 \%$ of radiotherapy treatments are now being delivered with IMRT ahead of the $24 \%$ target.

The cancer taskforce's report includes various recommendations specific to screening programs and radiotherapy technology, and the challenge will be to meet these over the next 5 years. By identifying these priorities now, we will look into proceeding to make the necessary improvements as quickly as possible.

\section{- Jane Maher}

We need to ensure we are supporting people living with cancer now. We also need to recognize that new treatments, while desperately needed, particularly for rarer cancers, do not necessarily have fewer side effects or long-term consequences than existing treatments. It is important to remember that ensuring the patients receive tried and tested treatments is also a priority if the UK population is to have as good outcomes as comparable countries in Europe.

\section{Q How important is utilizing routine data \& cancer registries? \\ - Jane Maher}

This is essential. We want people with cancer in the UK to experience the very best care and support because the health and social care system understands and responds to what matters to them. Routinely collected data have a major role to play in achieving this. Macmillan works closely with Public Health England's National Cancer Intelligence Network and cancer registries across the UK to develop and apply insight from routine data. However, we have to couple the collection and use of data with strong data governance and safeguards that allow timely and safe sharing of data, and enable people with long-term conditions, the general public and healthcare professionals to trust and support this approach.

\section{- Gina Radford}

Utilizing routine data and cancer registries is vital as they tell us a great deal (whether they relate to incidence, mortality and survival statistics, waiting times, CCG performance or patient experience). They allow us to build up a picture of what the current situation is, where we are improving and where there is scope to improve still further. Success in delivering the aspirations of the strategy will depend and rely on the intelligent use of data to drive continuous improvement. Indeed, many of the recommendations focus on the key role of data and metrics specifically.

\section{Q How is the funding \& eligibility for}

high-cost cancer treatments changing in the UK?

\section{- Gina Radford}

It is crucial to provide cancer patients with access to the latest radiotherapy treatments and a great deal is being done in this regard. In 2013, a $£ 23$ million fund was used to improve access to IMRT.

In January 2015, NHS England announced the extension of its commissioning through evaluation program to stereotactic ablative radiotherapy (SABR) costing in the region of $\mathrm{GB} £ 5$ million each year, for 3 years. NHS England's evaluation program will enable the number of cancer patients eligible to access SABR treatment to rise significantly, by 750 new patients a year and will widen the number of cancers being treated. The investment is in addition to NHS England's pledge to fund up to $£ 6$ million over the next 5 years to cover the NHS treatment costs of SABR clinical trials led largely by Cancer Research UK.

In 2012, the Government provided GB£250 million to build two proton beam therapy centers in England (at University College London Hospital and The Christie). Work is currently underway and the first of these centers will be fully operational in 2018. Currently, the NHS 
funds treatment abroad for patients in certain categories, so being able to have this treatment in England in the future is a great positive.

With reference to the Cancer Drugs Fund, since October 2010, it has helped over 84,000 cancer patients in England. The fund was increased to GB£280 million for 2014/15 and for this year NHS England has made available a further $\mathrm{GB} £ 60$ million bringing the total to GB£340 million, to help more cancer patients access the life-extending drugs that they need.

\section{- Jane Maher}

The high cost of cancer treatment is a major challenge for all of us and particularly the NHS, government and pharmaceutical companies. In England, the Cancer Drugs Fund was never intended to be a long-term solution and the recent decision to remove some of the drugs from the list reflects that it is under strain and may not be sustainable. We are worried about the impact this situation will have on people with cancer. The alternatives in Scotland and northern Ireland still need to prove that they match the access to drugs available elsewhere. Decision makers in the NHS from across the UK must work together with charities to find an alternative system which will make sure that no matter where a person lives, how old they are or what type of cancer they have, they are able to get timely access to cancer treatment that is right for them.

\section{Q How do you believe we can bend the cancer cost-curve? \\ - Gina Radford}

The cost of cancer in the NHS is likely to grow rapidly due to increasing incidence, healthcare inflation and new technology. Furthermore, we have to consider the actions and initiatives outlined in the report, which will require additional investment, including one-off capital costs. In the longer term, savings can be made if we are able to prevent more cancers, diagnose people earlier, and provide innovative and cost-effective treatment, which can in turn offer improved quality of life for patients and reduced NHS costs in the long term.

\section{- Jane Maher}

Investing in the right way now can unlock efficiency savings for the NHS. In the medium term, fully funding the implementation of cancer strategies across all four of the UK nations should contribute savings substantially in excess of their annual cost. Interventions such as the recovery package and stratified follow-up pathways improve value by reducing the number of outpatient appointments and deploying resource more effectively to provide more personalized care. To improve cancer outcomes, there must be investment across the whole cancer pathway, including post-treatment.

\section{How can we coordinate what we know at a national level to create an international oncology framework? \\ - Gina Radford}

We can learn a great deal from international comparisons and benchmarking. This is the case with survival statistics in terms of the cancers where we are narrowing the gap with the best performing countries, and those where we still have greater progress to make. In addition, by looking at our way of working in areas as diverse as public awareness; GP referral; pathways of care; treatment; research and workforce/recruitment, we can have a better idea of where we are on an international scale.

\section{- Jane Maher}

We need to create expert communities of influence at local, national and international levels. This includes international research programs - for example, Macmillan funds a small part of the International Cancer Benchmarking Partnership - and international conferences, such as the World Cancer Congress. Cancer is a leading health challenge in the majority of developed countries, but comparisons of international survival rates show that some countries are responding to this challenge more effectively than others. It is also an increasing challenge for developing countries. We need to share examples of best practice and innovative solutions to improve care for all people with cancer.

Q Finally, where would you like to see cancer services in 10 years' time?

\section{- Jane Maher}

We want to see more people living longer following a cancer diagnosis, but in addition to that, much stronger emphasis on promoting recovery and improving quality of life so people can live healthy lives out of hospital and have a good experience at all stages of the pathway. We want to see major improvements in quality of care and experience, particularly at the end of life. 
We want care to be holistic and personalized for all people living with cancer. To enable this to happen we need to see redesigned models of care, a flexible workforce, effective use of data to drive service improvement and a harnessing of the potential of digital technology.

\section{- Gina Radford}

The new strategy estimates that if the NHS is successful in implementing the initiatives and ambitions outlined in its priorities and recommendations, there will be an additional 30,000 patients per year surviving cancer for more than 10 years or more by 2020, 11,000 through early diagnosis. There will also be a closing in the gap in survival rates between England and the best countries in Europe and elsewhere. We know that the incidence of cancer will continue to rise, due in part to an aging population, but by focusing on prevention, raising awareness and promoting early diagnosis, as well as investing in new technology and treatments, we will put ourselves in a good position in terms of improving cancer services and outcomes.

\section{Acknowledgements}

Professor Jane Maher thanks colleagues at Macmillan for their helpful input into this article.

\section{Disclaimer}

The opinions expressed in this article are those of the author and do not necessarily reflect the views of Future Medicine Ltd.

Financial \& competing interests disclosure

$J$ Maher and $G$ Radford have no relevant affliations or financial involvement with any organization or entity with a financial interest in or financial conflict with the subject matter or materials discussed in the manuscript. This includes employment, consultancies, honoraria, stock ownership or options, expert testimony, grants or patents received or pending, or royalties.

No writing assistance was utilized in the production of this manuscript. 